\title{
PET/CT in Clinical Practice
}

T.B. Lynch

New York, NY: Springer, 2007, 241 pages, \$39.95

PET/CT has an ability that has revolutionized the detection and staging of various tumors: the ability to accurately identify the anatomic location of abnormal metabolic activity. This new technology has also had a significant impact on cardiology and neurology.

This small handbook of 10 chapters is clearly written and focuses on the role of PET/CT in lung cancer, lymphoma, esophageal and gastric cancers, colorectal cancer, head and neck cancers, melanoma, and cancers of the male and female reproductive systems. Each chapter outlines the relevant staging system and includes illustrations to point out how PET/CT can best be deployed in cancer management. The 241 figures are of uniformly high quality and enhance the message of the text. Chapter 1 provides a general introduction to cellular biology, chapter 10 is a brief overview of the physics of PET/CT, and chapter 9 deals with normal PET/CT findings and common variants.

This handy book is a starting point for anyone interested in learning a little about PET/CT. The emphasis throughout has been to provide easy-to-read and accessible information. However, the entire range of available positron emitters is not considered, the focus being only on ${ }^{18} \mathrm{~F}-$ FDG.

This book is concise, relevant, superbly illustrated, and useful for trainees and practitioners in radiology, nuclear medicine, and oncology. 